\title{
Optimized Silicon Antiresonant Reflecting Optical Waveguides for Sensing Applications
}

\author{
F. Prieto, L. M. Lechuga, A. Calle, A. Llobera, and C. Domínguez
}

\begin{abstract}
The development of an evanescent field sensor with an integrated Mach-Zehnder interferometric (MZI) configuration requires the fabrication of optical waveguides with two main characteristics: 1) monomode behavior and 2) high surface sensitivity for sensing biomolecular interactions in a direct way (without labels). In this paper, we present an experimental study for the optimization of the different parameters of the waveguides that will be the basis of a highly sensitive optical sensor. After optimization, an MZI sensor has been fabricated and some sensing applications are shown. The designed waveguides are based on antiresonant reflecting optical waveguide (ARROW) structures and are fabricated with standard silicon technology.
\end{abstract}

Index Terms-Antiresonant reflecting optical waveguide (ARROW) theoretical design, evanescent wave sensors, Mach-Zehnder interferometer (MZI), silicon technology.

\section{INTRODUCTION}

I NTEGRATED optical devices are increasingly being used as transducers for optochemical sensing applications [1]. The utilization of microtechnology for the integration of these devices offers some advantages as a better control of the light path by the use of optical waveguides, mechanical stability, higher sensitivity, miniaturization, and the possibility of mass-production.

In general, integrated optical sensors make use of the evanescent field detection principle. In an optical waveguide, light travels confined within the core layer. However, part of the guided mode (evanescent field) travels through a region that extends outward, around $100 \mathrm{~nm}$, into the media surrounding the waveguide. When there is a change in the optical characteristics of the outer medium (i.e., refractive index change), a modification of the optical properties of the guided mode is induced via the evanescent field. To detect this variation, several transduction schemes can be used, such as resonant mirror, grating coupler, etc. [2]-[4]. We have chosen an interferometric method based on the Mach-Zehnder (MZ) configuration [5] due to its higher sensitivity compared to other schemes [6], [7]. In this sensor configuration, the refractive index change is evaluated by the intensity modulation produced

Manuscript received May 9, 2000; revised October 5, 2000. This work was supported by the Comisión Interministerial de Ciencia y Tecnología (Projects AMB98-1048-C04-02, TIC97-0594-C04-01) and by the Comunidad Autónoma de Madrid (C.A.M.) (Project 07M/0051/1999),

F. Prieto, L. M. Lechuga, and A. Calle are with the National Center of Microelectronics (IMM-CSIC), 28760 Tres Cantos, Madrid, Spain (e-mail cnmar03@fresno.csic.es).

A. Llobera and C. Domínguez are with the National Center of Microelectronics (IMB-CSIC), 08193 Bellaterra, Barcelona, Spain.

Publisher Item Identifier S 0733-8724(01)00399-1. by the interference of light travelling through the two arms of the interferometer, one of which is exposed to the refractive index variations of the outer medium.

To obtain an efficient transducer for detecting molecular interactions, the optical waveguides of the sensor should have high surface sensitivity. The strength and distribution of the evanescent field in the outer medium need to be maximized in order to assure a high response for changes in the optical properties of the surrounding medium. In particular, the sensor is being developed for the detection of biochemical reactions between a receptor molecule and its complementary analyte. This bio-interaction takes place on the waveguide surface and the size of the involved elements is around a few nanometers, much less than the field penetration into the outer medium $(100 \mathrm{~nm}$ for wavelengths in the visible). For that reason, it is very important to maximize the power value of the guided mode at the core-cover interface, that is, the surface sensitivity [8], [9]. For example, to measure in a direct way a bio-interaction that involves a change in the molecular layer thickness of $0.01 \mathrm{~nm}$, the sensor should detect a change of the effective refractive index of $\Delta N \approx 4 \cdot 10^{-6}[8]$.

Optical waveguides based on Total Internal Reflection (TIR) have been experimentally proved to have high surface sensitivity [10]. For implementation in a Mach-Zehnder Interferometer (MZI), these waveguides should have monomode behavior that, assuming a fixed wavelength, can be controlled with a proper design of the thickness and refractive indexes of the waveguide layers. Finally, a rib structure is designed to achieve lateral light confinement. Width and depth of this rib are also essential for assuring a single-mode optical waveguide. A monomode TIR waveguide can be designed with a core thickness of several micrometers if the refractive index difference between core and cladding layers is small (less than 0.05). In this case, the latter is required to be thick enough (typically of several micrometers) to reduce the radiation loss due to the penetration of the evanescent field into the substrate. In the fabrication process, this means a longer time to form the thick cladding layer and the need to control accurately the refractive index. Depending on the thickness of the final structure, a cracking of the multilayer structure can occur if the stress of each single layer is not controlled. Other alternative is to design a core thickness in the order of hundreds of nanometers, if the refractive index difference between the core and the cladding layer is high enough (more than 0.1). In this way, the cladding layer thickness can be decreased to a few micrometers due to the small penetration of the evanescent field into the cladding. However, the rib depth must be around several nanometers for single-mode TIR waveguides (an integrated MZI sensor based on $\mathrm{Si}_{3} \mathrm{~N}_{4}$ waveguides with a core thickness of 
around $100 \mathrm{~nm}$ and a rib depth of $3 \mathrm{~nm}$ has been previously described [11]). These small dimensions are a technological drawback for fabrication and mass-production of sensors based on monomode TIR waveguides.

To overcome this problem we have studied the possibility of using ARROW structures. The most important characteristic of these waveguides is their single-mode behavior, even for core dimensions of several micrometers and rib parameters of a few micrometers, together with a cladding thickness even smaller than the core thickness. This means technological and experimental advantages: the relatively large core size is suitable for efficient connection to single-mode commercial optical fibers.

In this paper, we show the fabrication and characterization of different types of ARROW structures focused on sensing applications, which theoretical design we have published before [12]. We will discuss their advantages and drawbacks to finally propose a structure that will be the basis of a highly sensitive optical MZ sensor. We will also present some examples of the designed interferometer as sensor.

\section{FABRICATION OF THE OPTICAL WAVEGUIDES}

The ARROW structure has been widely studied [13]-[15]. The ARROW is a special multilayer waveguide where light is confined within the core by TIR at the core-outer medium interface and by an antiresonant reflection, with a very high reflectivity of $99.96 \%$, at the two interference cladding layers underneath the core. With these two layers virtual single-mode behavior is obtained by loss discrimination of modes higher than the fundamental. The basic characteristics of these waveguides are: low losses for the fundamental mode, large light confinement, high tolerance for the design of the refractive indexes and thickness of the cladding layers.

Several materials can be used for the different layers of the ARROW [14], [16]. We have based the fabrication of the structures in silicon technology using materials with no optical absorption in the visible range, at which the biomolecules to be detected are transparent (around $633 \mathrm{~nm}$ ). These materials are, basically, silicon oxide and silicon nitride. Moreover, the use of this well-known technology will allow the integration of sources, sensors, detectors and amplifier circuitry on the same chip, important subject for mass-production in the future.

The two ARROW structures that have been chosen for their implementation in the MZI are 1) ARROW-A and 2) ARROW-B [17]. They differ in the value of the refractive index of the first cladding layer. For the ARROW-A type [Fig. 1(b)] this value $\left(n_{1}=2.00\right)$ is higher than those of the core and second cladding $\left(n_{\text {core }}=1.485\right.$ and $n_{2}=1.46$, respectively), while for the ARROW-B type [Fig. 1(a)], the refractive index of the first cladding is lower $\left(n_{1}=1.46\right.$ compared to $n_{\text {core }}=n_{2}=1.54$ ). Another difference between these structures is that the ARROW-A type is polarization selective (TE polarized light experiences lower losses than TM polarized light by the same phenomenon which gives rise to the Brewster angle) and the ARROW-B type is less dependent on polarization.

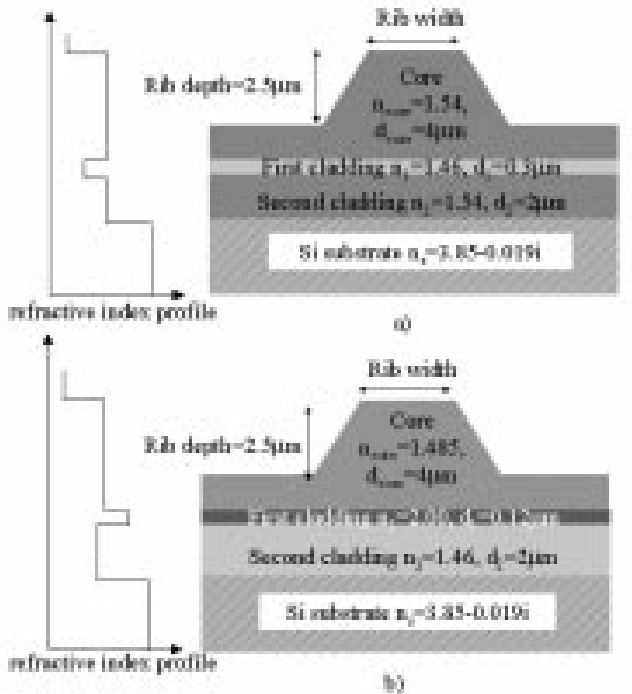

Fig. 1. Channel waveguide structure and refractive index profile of (a) ARROW-B and (b) ARROW-A.

For the design of the ARROW structures, a homemade computational program based on the Non-Uniform Finite Difference Method [12], [18] has been developed. For a wavelength of $632.8 \mathrm{~nm}$, the effective refractive index and attenuation losses were evaluated for several ARROW-A and ARROW-B structures varying the refractive indexes and thickness of the different ARROW layers. For a detailed discussion of the calculations, see [12] and [15]. With the results obtained in both references, we fabricated the two different ARROW structures described in Fig. 1.

The first one is an ARROW-B type with a core refractive index of 1.54 and a thickness of $4 \mu \mathrm{m}$; the first cladding has a refractive index and thickness of 1.46 and $0.3 \mu \mathrm{m}$, respectively; and the second cladding refractive index is 1.54 , while its thickness is $d_{\text {core }} / 2$, i.e., $2 \mu \mathrm{m}$.

The second structure is an ARROW-A type with a core refractive index of 1.485 and a thickness of $4 \mu \mathrm{m}$. The refractive index and thickness of the first cladding are 2 and $0.12 \mu \mathrm{m}$, respectively. Finally, the second cladding refractive index is 1.46 while its thickness is $d_{\text {core }} / 2$, i.e., $2 \mu \mathrm{m}$. This structure is slightly different from the original ARROW-A where the refractive indexes of the core and second cladding layers are equal [13]. The reason for this asymmetric structure is that, for sensor applications, the MZI has to be locally protected with a covering layer (with a refractive index of 1.46). To confine light in the core layer its refractive index has to be higher (i.e., 1.485). This new structure is less dependent on polarization and presents lower attenuation losses. A drawback is that it loses the virtual single-mode behavior, being possible the propagation of several higher order modes for certain excitation conditions. Therefore, the proper design of the waveguide parameters (rib, depth, and width) is of crucial importance for obtaining a monomode structure [19].

The fabrication process is similar in both cases. ARROW structures are fabricated in our Clean Room facilities using a silicon CMOS compatible process [20]. A p-(100) silicon wafer is used as substrate. The second cladding is a $2-\mu \mathrm{m}$ silicon oxide layer grown by Thermal Oxidation of the substrate for 


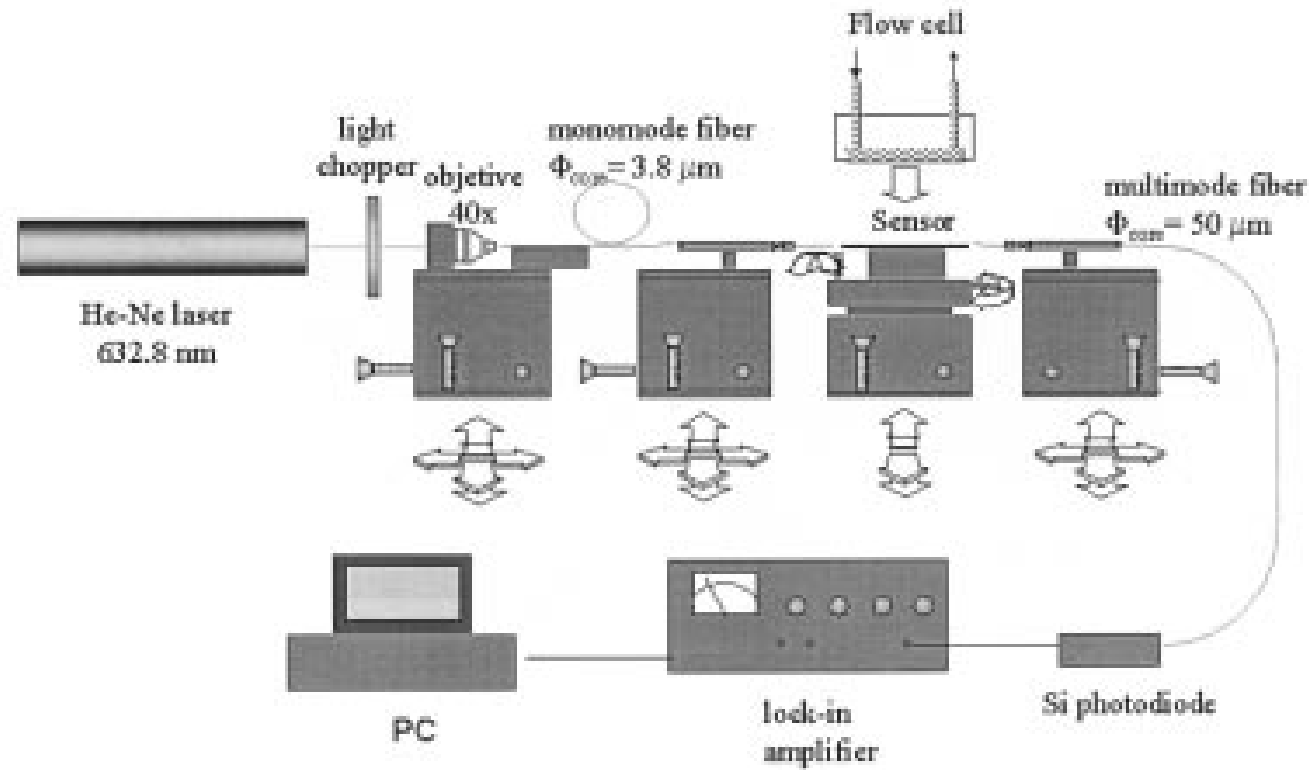

Fig. 2. Experimental setup.

the ARROW-A type $\left(n_{2}=1.46\right)$, or deposited by plasma enhanced chemical vapor deposition (PECVD) at $300^{\circ} \mathrm{C}$ for the ARROW-B type $\left(n_{2}=1.54\right)$. The technology of growing nonstoichiometric silicon oxide $\left(\mathrm{SiO}_{x}\right)$ by PECVD has been developed previously in our group [21] and it is possible to vary the refractive index between 1.46 and 1.72. The first cladding is, for the ARROW-A type, a $0.12-\mu \mathrm{m}$ silicon nitride $\left(\mathrm{Si}_{3} \mathrm{~N}_{4}\right)$ layer deposited by low pressure chemical vapor deposition (LPCVD) at $800{ }^{\circ} \mathrm{C}\left(n_{1}=2.00\right)$. For the ARROW-B type, the first cladding is a $0.3-\mu \mathrm{m}$ PECVD silicon oxide layer $\left(n_{1}=1.46\right)$. After each layer deposition, the refractive index and thickness are tested by ellipsometry.

To obtain lateral confinement of light, a rib structure is fabricated. The rib depth was designed to be around $60 \%$ of the core thickness $(2.5 \mu \mathrm{m})$ to obtain good confinement of light. Several samples were designed with different widths, ranging from 1 to $40 \mu \mathrm{m}$, to experimentally analyze its influence in the guiding properties of the structure. The rib is defined on the core layer by reactive ion etching (RIE) (Fig. 1).

For the experimental study of the optical characteristics of the waveguides (attenuation losses, insertion losses, field distribution), the processed wafer is cut in individual pieces of different lengths. To efficiently couple light into the waveguides, the input and output edges of each piece are polished to produce a scratch- and damage-free surface. This process is accomplished by using lapping film sheets of different silicon carbide ( $\mathrm{SiC}$ ) grain size until obtaining an edge surface with optical quality.

\section{Comparison BetweEn Arrow-A AND Arrow-B STRUCTURES}

A schematic of the experimental set-up used is shown in Fig. 2. Light from a He-Ne laser $(\lambda=632.8 \mathrm{~nm})$ is coupled to a single-mode optical fiber $(3.8 \mu \mathrm{m}$ core diameter) using a microscope objective $(40 \times)$. The end of the monomode fiber is placed in front of the waveguide rib face to couple light into the ARROW structure (end-fire coupling). Light is collected by a multimode standard optical fiber ( $50 \mu \mathrm{m}$ core diameter) connected to a silicon photodiode. Precise translation stages are used for the accurate alignment of all the components. A synchronous detection scheme is used with the aid of a lock-in amplifier and a light chopper. Alternatively, a microscope objective $(100 \times)$ and a silicon CCD camera are used to study the field distribution at the end of the waveguide.

First, an experimental study was made to analyze the guiding conditions of the rib ARROW-A and B structures. Propagation losses at $632.8 \mathrm{~nm}$ as a function of the waveguide width were measured by the cutback method using samples with different lengths. Measurements were performed with TE and TM-polarized light launched into a mono-mode optical fiber with a small length (around $15 \mathrm{~cm}$ ) that was placed in straight line to preserve the input polarization.

Fig. 3 shows total losses versus rib width for the ARROW-A and ARROW-B structures with a core thickness of $4 \mu \mathrm{m}$, a rib depth of $2.5 \mu \mathrm{m}$, and a length of $1 \mathrm{~cm}$. For the ARROW-B type, propagation losses for ribs wider than $10 \mu \mathrm{m}$ are about $0.2 \mathrm{~dB} / \mathrm{cm}$ with insertion losses of $0.9 \mathrm{~dB}$ (see Fig. 4). In the case of the ARROW-A type, total losses include attenuation of $0.4 \mathrm{~dB} / \mathrm{cm}$ and insertion losses of $1.7 \mathrm{~dB}$. In both cases, for rib widths lower than $10 \mu \mathrm{m}$, losses show a sharp increase produced by the loss discrimination of modes higher than the fundamental and by the higher insertion losses when the rib width is closer to the core diameter of the input fiber $(3.8 \mu \mathrm{m})$.

If we analyze the field distribution at the end of the waveguide [Fig. 3(b) and (c)], we observe horizontal multimode behavior for ribs wider than $8 \mu \mathrm{m}$, while for rib widths smaller than 8 $\mu \mathrm{m}$ only the fundamental mode is supported. We can also observe, for noncentred excitation conditions, a bimodal distribution in the vertical direction for the ARROW-A with rib widths 


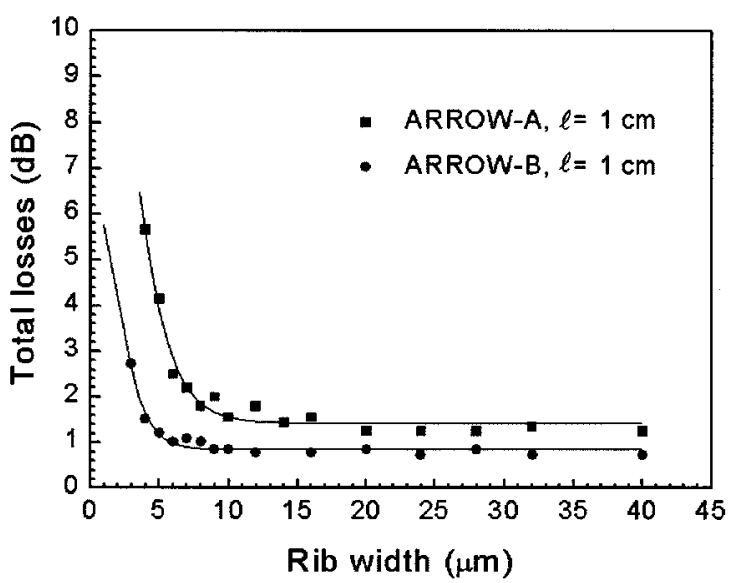

(a)

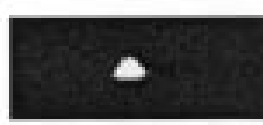

$w=6 \mu \mathrm{m}$

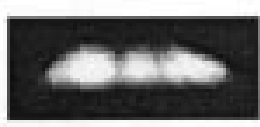

$w=20 \mu \mathrm{m}$

(b)

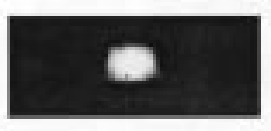

$w=6 \mu m$

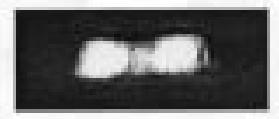

$\mathrm{w}=14 \mu \mathrm{m}$

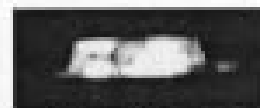

$\mathrm{w}=14 \mu \mathrm{m}$ (c)

Fig. 3. (a) Attenuation (TE polarization) versus rib width for two different ARROWs: 1) ARROW-A type and 2) ARROW-B type. Experimental field distribution for two different ARROW structures: (b) ARROW-B and (c) ARROW-A.

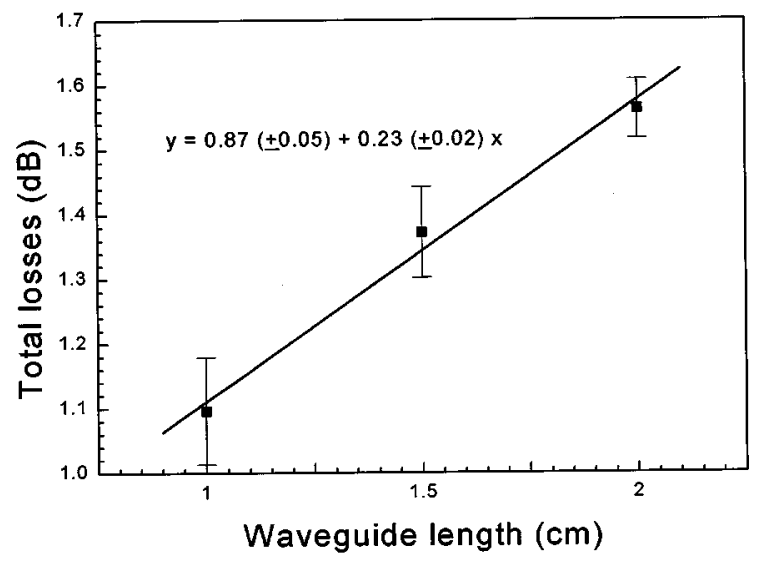

Fig. 4. Measurement of propagation losses for ARROW-B by the cutback method. The slope from the linear fitting is related to the propagation losses, while the value at the ordinate axis shows the coupling losses.

larger than $14 \mu \mathrm{m}$. This multimode behavior disappears for narrower waveguides due to the increase in the attenuation losses for higher order modes.

Attenuation losses as a function of the wavelength and polarization were measured for an ARROW-B structure with a rib width of $40 \mu \mathrm{m}$. Measurements were performed using a Na lamp

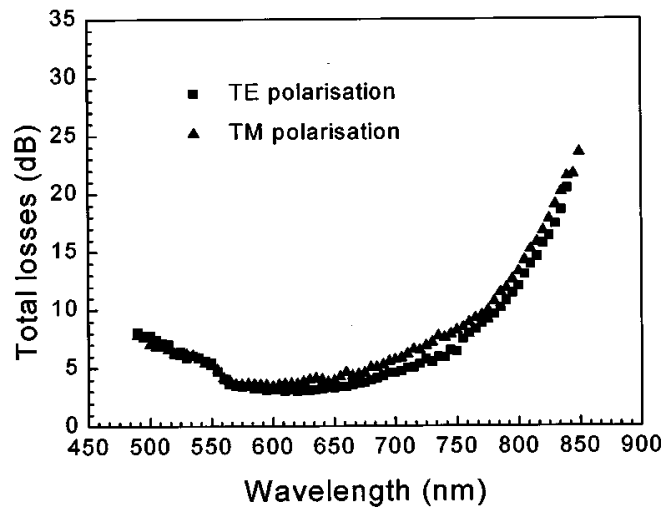

Fig. 5. Attenuation as a function of wavelength for TE and TM polarization. Measurements performed over a $1 \mathrm{~cm}$ long ARROW-B structure.

and a Jobin Ybon Spex monochromator. Light was launched directly into the waveguide by end-fire coupling with a microscope objective $(40 \times)$. A linear polarizer was placed before the objective to select polarization. Results are shown in Fig. 5, where we can observe a minimum in the attenuation around the designed wavelength $(632.8 \mathrm{~nm})$. Attenuation differences for the TE and TM polarization are small as is expected from the behavior of an ARROW-B structure.

For the ARROW-A structure, measurements varying the input wavelength were previously presented [19]. We did not appreciate any difference between TE and TM polarized light due to the high core refractive index $\left(n_{\text {core }}=1.485\right)$, which implies a low dependence on polarization, as it was stated above.

\section{ARrow FOR SENSING APPLICATIONS}

For sensing purposes, the optical waveguide structures are designed to assure single mode behavior and high surface sensitivity, what means that the sensor response for changes in the optical properties of the outer medium must be as high as possible. Surface sensitivity, calculated for processes that involve the adsorption of molecules from a gaseous or liquid phase, is defined as the rate of change of the effective refractive index of the guided mode, $N$, as the thickness of the homogeneous molecular adlayer, $d_{\ell}$, varies [8], [9]. This sensitivity is related to the squared field magnitude of the guide mode at the core-outer medium interface, considering a homogeneous adsorbed layer of refractive index $n_{\ell}$ and thickness $d_{\ell}$. We have previously developed the analytical expression of surface sensitivity for an ARROW structure in reference [12].

In the fabricated waveguides, the optical mode is highly confined within the core layer and there is a small penetration of the evanescent field into the outer medium (around $100 \mathrm{~nm}$ ) (Fig. 6). For these structures, surface sensitivity is very low. One possibility to increase the evanescent field penetration into the outer medium is to decrease the core refractive index. For that, ARROW-A type is more advantageous than the ARROW-B structure due to its lower core refractive index (Fig. 6).

Another way to increase sensitivity is to diminish the core thickness (Fig. 7). In the original ARROW-A structure, the 


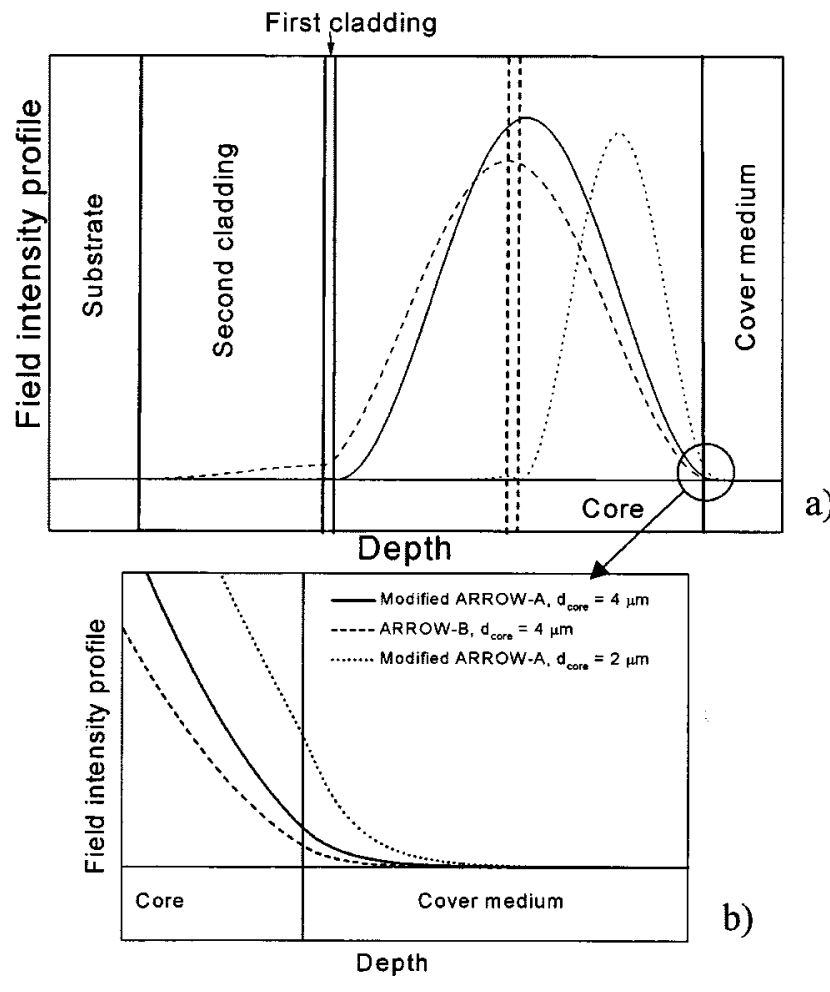

Fig. 6. (a) Intensity field profile (TE polarization) for the modified ARROW-A (with two different core thickness) and the ARROW-B. (b) Detail of the evanescent field for the three structures. Field penetration is higher for the ARROW-A with a core thickness of $2 \mu \mathrm{m}$, which corresponds to a higher sensitivity.

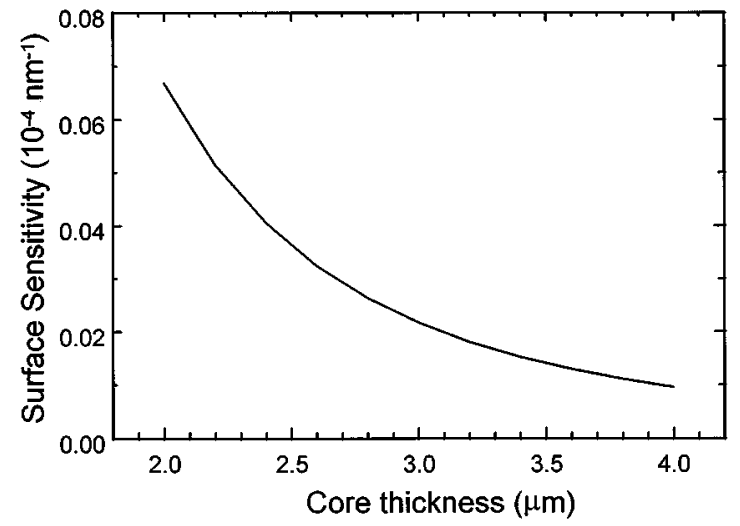

Fig. 7. Surface sensitivity as a function of the core thickness for the ARROW-A structure.

second cladding layer thickness is kept half of the core. As the core thickness diminishes, light is guided closer to the substrate, with the subsequently increase of the attenuation losses due to the high absorption of silicon in the visible part of the spectrum. We propose two alternatives to overcome this problem: 1) a modified multi-ARROW-A structure (Fig. 8) with two pairs of interference cladding; and 2) an ARROW-A structure modified by maintaining fixed the thickness and refractive index of the second cladding in $2 \mu \mathrm{m}$ and 1.46 , respectively.

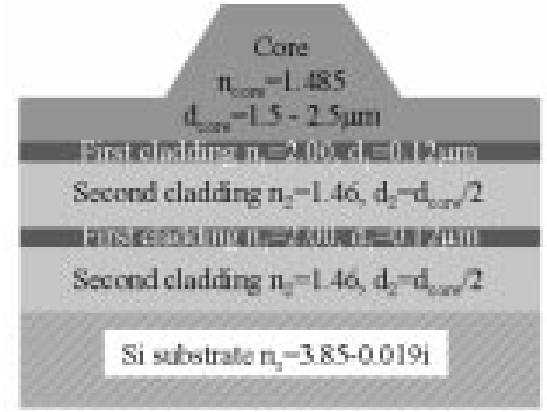

Fig. 8. Rib multi-ARROW-A structure.

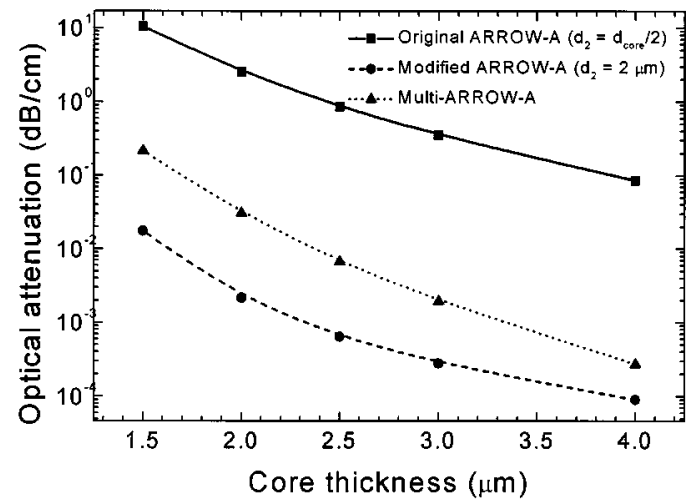

Fig. 9. Attenuation versus core thickness. Theoretical comparison between the original ARROW-A structure $\left(n_{\text {core }}=1.46, d_{2}=d_{\text {corc }} / 2\right)$, the modified ARROW-A $\left(n_{\text {core }}=1.485, d_{2}=2 \mu \mathrm{m}\right)$ and the multi ARROW-A.

\section{A. MULTI-ARROW-A:}

With the inclusion of an additional pair of interference cladding layers, attenuation decreases theoretically between one and two orders of magnitude, depending on the core thickness (Fig. 9). However, from the fabrication point of view, we increase the mechanical stress of the multilayer system by adding these two new layers, what could lead to the peel-off of the cladding layers, as occurred in some of the processed structures. We have fabricated multi-ARROW-A structures with a core thickness of 1.5 and $2.5 \mu \mathrm{m}$ to assure high surface sensitivity. The second cladding of the interference layers was maintained in $d_{\text {core }} / 2$ and the rib width designed was 8 and $10 \mu \mathrm{m}$ to obtain lateral single-mode behavior. Although theoretically these structures have attenuation losses below $1 \mathrm{~dB} / \mathrm{cm}$ (Fig. 9), experimentally they are not useful for sensor applications due to their high losses. These include, not only high insertion losses (around $10 \mathrm{~dB}$ ), but also high attenuation (close to $15 \mathrm{~dB} / \mathrm{cm}$ ) produced by a guiding deficiency due to the thin cladding layers. The proposed multi-ARROW-A structures are not a good alternative due to their high losses together with the more complexity in their fabrication process.

\section{B. MODIFIED ARROW-A}

About the second alternative (a modified ARROW structure with a fixed $2-\mu \mathrm{m}$ second cladding of refractive index 1.46) we have processed waveguides with core thickness of $1.5,2$, and $3 \mu \mathrm{m}$ and with rib widths ranging from 1 to $40 \mu \mathrm{m}$ to analyze their attenuation losses and modal behavior. The characteristics 
of the structures are: a second cladding with a thickness of $d_{2}=$ $2 \mu \mathrm{m}$ and a refractive index of $n_{2}=1.46$; a first cladding layer with $d_{1}=0.12 \mu \mathrm{m}$ and $n_{1}=2.00$; a core layer with a refractive index of $n_{\text {core }}=1.485$ and thickness of $3 \mu \mathrm{m}$ (with a rib depth of $1.9 \mu \mathrm{m}$ ), $2 \mu \mathrm{m}$ (with a rib depth of $1.2 \mu \mathrm{m}$ ) and $1.5 \mu \mathrm{m}$ (with a rib depth of $1.2 \mu \mathrm{m})$.

Results are shown in Fig. 10 for TE and TM polarization. For waveguides with a core thickness of $3 \mu \mathrm{m}$, results are similar to the ARROW-A structures with $d_{\text {core }}=4 \mu \mathrm{m}$ [Fig. 3(a)]. Attenuation losses for ribs wider than $7 \mu \mathrm{m}$ are around $0.4 \mathrm{~dB} / \mathrm{cm}$ for a core thickness of $3 \mu \mathrm{m}$ with insertion losses in the order of $2 \mathrm{~dB}$ [Fig. 10(a)]. Losses increases for rib widths smaller than $7 \mu \mathrm{m}$ due to loss discrimination of modes higher than the fundamental. The field distribution profile shows monomode behavior for rib widths lower than $7 \mu \mathrm{m}$ [Fig. 10(b)]. We do not appreciate any difference between losses for TE or TM polarization.

For waveguides with a core thickness of $2 \mu \mathrm{m}$, attenuation losses are around $0.5 \mathrm{~dB} / \mathrm{cm}$ for the TE polarization and $0.6 \mathrm{~dB} / \mathrm{cm}$ for TM polarization. As in the former case, losses increase for rib widths lower than $7 \mu \mathrm{m}$, the same width where waveguides start to show mono-mode behavior.

When the core thickness is $1.5 \mu \mathrm{m}$, attenuation for waveguides wider than $10 \mu \mathrm{m}$ is around $1.2 \mathrm{~dB} / \mathrm{cm}$, with insertion losses around $10 \mathrm{~dB}$. Losses increase sharply for widths lower than $10 \mu \mathrm{m}$ [Fig. 10(a)]. For these rib dimensions, we can also observe single-mode behavior [Fig. 10(c)]. Differences between attenuation for TE and TM modes are more appreciable when the rib width is lower than $10 \mu \mathrm{m}$.

\section{SENSOR CONFIGURATION}

ARROW channel structures are the basis of an integrated evanescent field sensor with an MZI configuration. In the integrated version of the MZI, an optical waveguide is split into two arms and after a certain distance they recombine again. The interferometer is covered with a protective layer and an area of length $L$ is opened in one of the arms (sensor arm) to bring into contact the waveguide and the environment. Only light travelling in the sensor arm will experience a phase shift induced by a change in the properties of the surrounding medium.

The protective layer must be compatible with Si technology. We deposit a silicon oxide layer with a refractive index of 1.46 by PECVD at $300{ }^{\circ} \mathrm{C}$. The thickness of this layer is $2 \mu \mathrm{m}$, enough to isolate the core from the environment. The opening of the sensor area in the protective layer is done, after a photolitographic process, by chemical etching with a $\mathrm{HF}: \mathrm{H}_{4} \mathrm{NF}$ (1:7) solution. If the etching process is not accurately controlled the $\mathrm{SiO}_{x}$ core layer can be also etched. Due to the inherently nonuniformity of the chemical etching process we must protect the core layer. This is done by inserting a new layer resistant to the etching solution between the protective layer and the core. This new cover is a silicon nitride $\left(\mathrm{Si}_{3} \mathrm{~N}_{4}\right)$ layer, deposited by LPCVD at $800^{\circ} \mathrm{C}$, with a thickness of $0.12 \mu \mathrm{m}$, analogous to the first cladding layer.

To evaluate the influence of these covering layers in the guiding characteristics of the waveguides, several samples with the modified ARROW-A structure and with protective layers of silicon nitride (thickness of $0.12 \mu \mathrm{m}$ ) and silicon

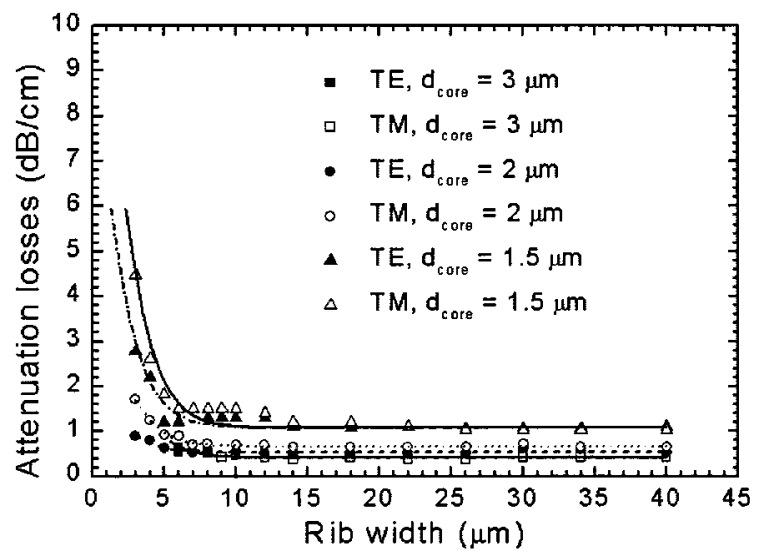

(b)
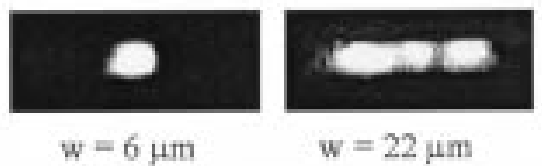

(b)
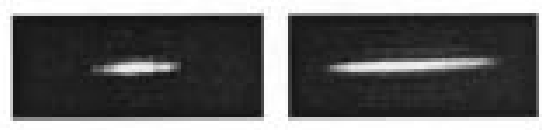

$w-22 \mu m$

(c)

Fig. 10. (a) Attenuation losses (TE and TM polarization) as a function of the rib width for an ARROW-A structure $\left(n_{\text {core }}=1.485, n_{2}=1.46, d_{2}=\right.$ $2 \mu \mathrm{m}$ ) with two different core thickness. Field distributions for two different rib widths: (b) core thickness of $3 \mu \mathrm{m}$ and (c) core thickness of $1.5 \mu \mathrm{m}$.

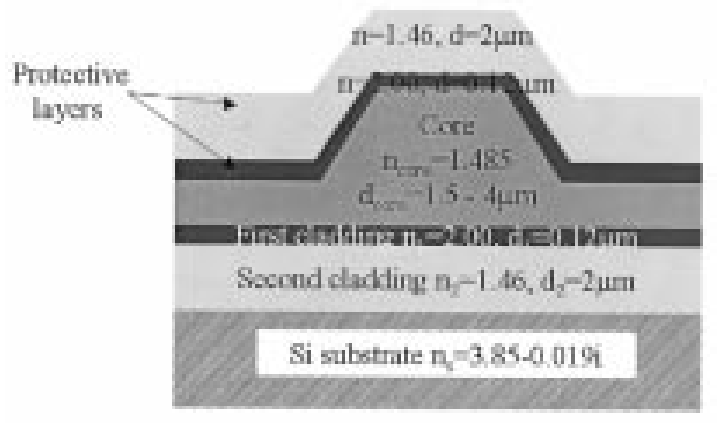

Fig. 11. Rib ARROW-A structure with protective layers.

oxide (thickness of $2 \mu \mathrm{m}$ ) were fabricated with different core thickness and rib widths (the structure is shown in Fig. 11), to compare with the noncovered waveguides. Results are shown in Fig. 12(a), where attenuation losses as a function of the rib width for TE polarization are shown.

When the core thickness is $4 \mu \mathrm{m}$, losses for rib widths wider than $15 \mu \mathrm{m}$ are due to an attenuation of about $0.5 \mathrm{~dB} / \mathrm{cm}$ and insertion losses close to $1 \mathrm{~dB}$. Insertion losses are lower because the optical mode from the input fiber is better adapted to the fundamental mode of the ARROW-A structure due to the silicon oxide covering layer. Losses show a sharp increase for rib widths lower than $15 \mu \mathrm{m}$. This value is slightly different from the noncovered waveguide case (around $10 \mu \mathrm{m}$ ) because, in general, attenuation increases with the presence of the protective 


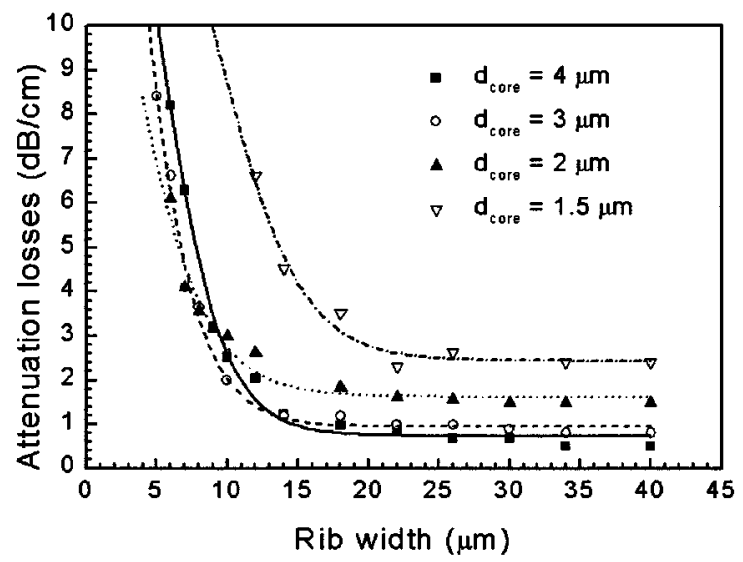

(a)

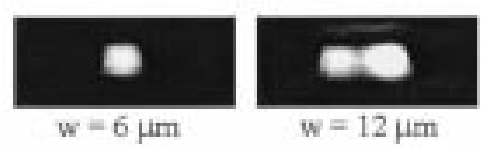

(b)

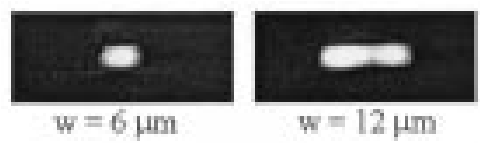

(c)
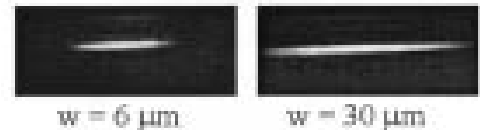

(d)

Fig. 12. (a) Attenuation losses (TE polarization) as a function of the rib width for an ARROW-A structure with covering layers $\left(n_{\text {core }}=1.485, n_{2}=1.46, d_{2}=2 \mu \mathrm{m}\right)$ for three different core thickness. Field distributions for two different rib widths: (b) core thickness of $4 \mu \mathrm{m}$; (c) core thickness of $3 \mu \mathrm{m}$; and (d) core thickness of $1.5 \mu \mathrm{m}$.

layers, being this increment more important for rib widths lower than $15 \mu \mathrm{m}$. We also observe monomode behavior for rib widths lower than $8 \mu \mathrm{m}$ [Fig. 12(b)].

When the core thickness is $3 \mu \mathrm{m}$, results are very similar. For ribs wider than $15 \mu \mathrm{m}$, attenuation is $0.8 \mathrm{~dB} / \mathrm{cm}$, with insertion losses close to $1 \mathrm{~dB}$. Single-mode behavior is also observed for rib widths lower than $8 \mu \mathrm{m}$ [Fig. 12(c)].

For waveguides with a core thickness of $2 \mu \mathrm{m}$, attenuation losses are around $1.5 \mathrm{~dB} / \mathrm{cm}$ with insertion losses of $4 \mathrm{~dB}$. Finally, for $1.5-\mu \mathrm{m}$ thick waveguides, attenuation is $2.4 \mathrm{~dB} / \mathrm{cm}$ for rib widths higher than $20 \mu \mathrm{m}$, with insertion losses around $8 \mathrm{~dB}$. We observe an increase in attenuation losses compared to the noncovered case, although insertion losses decrease [Fig. 12(a)]. The width where attenuation losses show a sharp increase is higher, although the single-mode behavior is observed for rib widths lower than $8 \mu \mathrm{m}$ [Fig. 12(d)].

Several MZI configurations were designed varying the separation between arms and the $\mathrm{Y}$-junction parameters. All the devices are symmetric with two different $\mathrm{Y}$-junction shapes: in the first case, the $\mathrm{Y}$-junction is formed with straight arms and opening angles of $1^{\circ}$ and $2^{\circ}$; in the second case, the Y-junction is shaped with circular bends with radii of 5 and $20 \mathrm{~mm}$.

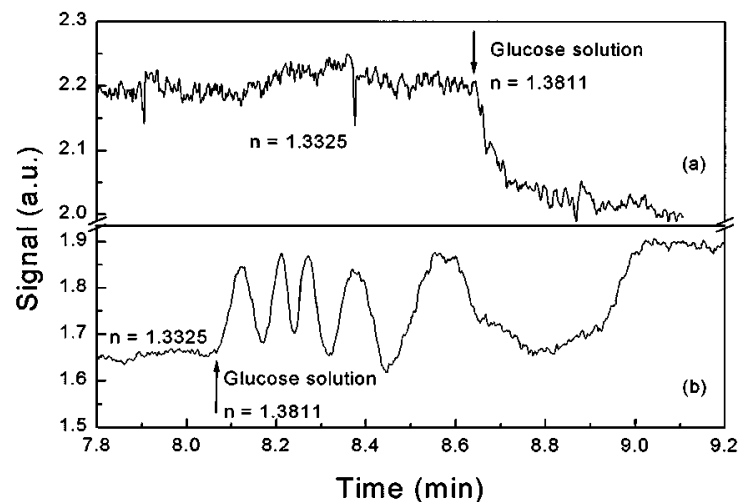

Fig. 13. Signal variation of the MZI as the refractive index in the sensor area changes as a function of time (TE polarization). Comparison when the silicon nitride overlay has a thickness of: (a) $120 \mathrm{~nm}$ and (b) $39 \mathrm{~nm}$.

In all cases, separation between the sensor and the reference arms exceeds $50 \mu \mathrm{m}$ to avoid coupling between modes travelling through both branches. In one arm, a $6 \mathrm{~mm}$ length and $100 \mu \mathrm{m}$ wide sensor area is created. The total length of the device is $35 \mathrm{~mm}$. Sensors are based on the waveguide structure described in Fig. 11, with a $4 \mu \mathrm{m}$ core thickness, an $8 \mu \mathrm{m}$ width, and with a silicon nitride protective layer of $0.12 \mu \mathrm{m}$. No differences in operation of the devices were observed and they were used interchangeably.

The sensor is being developed for the detection of biochemical interactions between a receptor molecule and its complementary analyte. The effect of this reaction is comparable to a change of the bulk refractive index of the outer medium. Therefore, the use of solutions with different refractive indexes is useful for studying the sensor sensitivity. Several solutions were prepared with different glucose concentrations in water, with refractive indexes varying from 1.3325 to $1.4004( \pm 0.0002)$, as determined by an Abbe refractometer operating at $25^{\circ} \mathrm{C}$. A flow cell mechanized in Teflon (with a channel width of $3 \mathrm{~mm}$, a depth of $100 \mu \mathrm{m}$, and a length of $15 \mathrm{~mm}$ ) is clamped onto the interferometer and a flow injection system is used to deliver the glucose solutions into the sensor area (see Fig. 2).

Sensitivity for the MZI used is expected to be low [12] due to the high confinement of the mode within the core. This can be corroborated in Fig. 13(a), where we show the signal variation at the sensor output as the bulk refractive index in the sensor area changes as a function of time.

\section{SENSITIVITY ENHANCEMENT}

As it has been shown previously [12], [22], sensitivity can be enhanced by overcoating the surface of the waveguide with high refractive index layers. If we use the silicon nitride protective layer, with a refractive index of 2.00 as the overlay, the theoretical thickness to obtain maximum sensitivity is around $d_{o v}=34 \mathrm{~nm}$ (see [12, Fig. 8]).

Therefore, we decided to etch the silicon nitride protective layer at the sensor area to a value close to $34 \mathrm{~nm}$. The etching was performed in $\mathrm{H}_{3} \mathrm{PO}_{4}$ bath at $180{ }^{\circ} \mathrm{C}$ (etch rate $10 \mathrm{~nm} / \mathrm{min}$ ). After the etching process, the thickness of the silicon nitride layer was diminished to, approximately, $39 \mathrm{~nm}$ (measured with a profilograph). Now, the sensor response clearly increases, as 


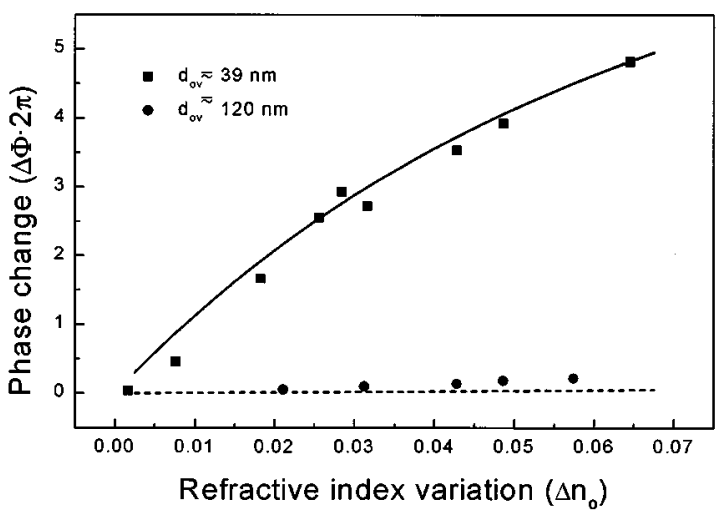

Fig. 14. Response of the MZI sensor as a function of the refractive index variation. The lines correspond to the theoretical simulation of the phase change: - (solid line) $d_{o v} \sim 39 \mathrm{~nm} ;-$ - (dashed line) $d_{o v} \sim 120 \mathrm{~nm}$.

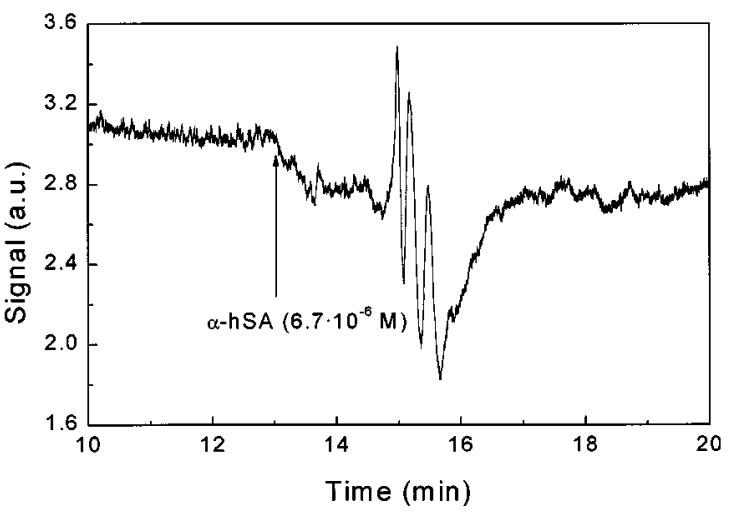

Fig. 15. Signal response (TE polarization) of the MZI sensor to the physical adsorption of a protein monolayer (commercial polyclonal antibody hSA).

can be seen in Fig. 13(b). In both cases (when the overlay thickness is $120 \mathrm{~nm}$ and $39 \mathrm{~nm}$ ), the MZI sensor was subjected to an exhaustive test by measuring the phase change as a function of the refractive index. The results from these measurements are presented in Fig. 14, where we can observe the sensitivity enhancement for an overlay thickness of $\sim 39 \mathrm{~nm}$. The lines in this graph correspond to the theoretical values and, as can be seen, the measured phase change is comparable to the model calculations [12].

As an example of biosensing application, in Fig. 15 it is shown the MZI sensor response to the physical adsorption of $6.7 \cdot 10^{-6} \mathrm{M}$ antihuman serum albumin protein $(\alpha$-hSA). Measurements are performed in phosphate buffered saline (PBS), with a pH of 7.35, at room temperature and maintaining a low flow rate $(33 \mu \mathrm{l} / \mathrm{min})$. Antibodies form a monolayer with a thickness of around $10 \mathrm{~nm}$ by physical binding (electrostatic forces) to the waveguide surface, originating a change in the effective refractive index of the guided mode and, therefore, in the MZI sensor output.

\section{CONCLUSION}

ARROW structures are promising waveguides for the development of high sensitive integrated optical sensors. Their fabrication process, based of standard silicon technology and the

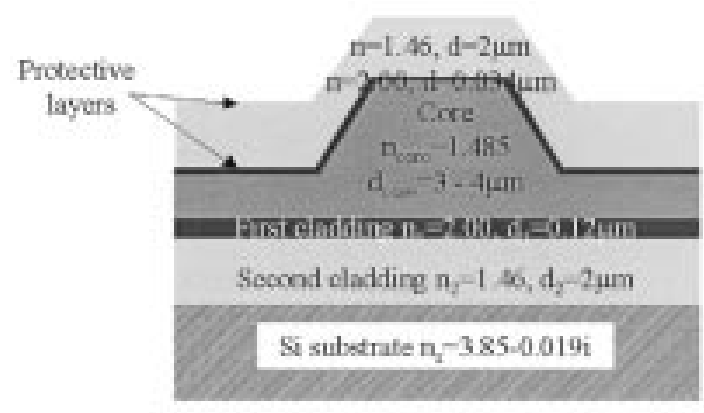

Fig. 16. Optimized ARROW-A structure for sensing applications.

large tolerance for the design of the refractive indexes and thickness of the waveguide layers, makes them very appropriate for low-cost mass production.

For their implementation in an optical sensor, based on the integrated MZI configuration, the optical waveguides must be covered with two protective layers. For this reason, the core refractive index should be higher than the silicon oxide-covering layer. These two covering layers affect the guiding properties of the ARROW structure, increasing the attenuation losses. In addition, the waveguides must verify two important conditions: single-mode operation and high surface sensitivity. To obtain monomode behavior, the core refractive index should be kept under a certain value, i.e., 1.485 , while the rib width has to be lower than $8 \mu \mathrm{m}$.

To assure high surface sensitivity, the core thickness can be diminished, with the corresponding increase of the attenuation losses. We have proposed two alternatives to overcome this problem. A multi-ARROW-A structure (with two pair of interference cladding layers) and an ARROW-A structure with a fixed second cladding layer thickness of $2 \mu \mathrm{m}$. This last alternative has been experimentally demonstrated to be more appropriate. Finally, sensitivity can also be increased by overcoating the waveguide surface with a high refractive index layer. The thickness of this overlay determines the enhancement factor. We have presented experimental results of a MZI sensor working as a refractometer where diminishing the overlay thickness to a value around $39 \mathrm{~nm}$, sensitivity is increased more than one order of magnitud.

In conclusion, we propose the optimized waveguide structure shown in Fig. 16 for sensing applications, which consists on a rib-ARROW-A structure with a silicon oxide core layer of $n_{\text {core }}=1.485$ and thickness higher than $2 \mu \mathrm{m}$; a silicon oxide second cladding layer with a refractive index of 1.46 and a fixed thickness of $2 \mu \mathrm{m}$ and a silicon nitride first cladding layer of thickness $0.12 \mu \mathrm{m}$. The waveguide is overcoated with a silicon nitride layer (of thickness around $34 \mathrm{~nm}$ ) and with a silicon oxide layer $(n=1.46)$ of thickness $2 \mu \mathrm{m}$. The rib depth is $60 \%$ of the core thickness and the rib width should be lower than 8 $\mu \mathrm{m}$ to obtain single-mode behavior.

\section{ACKNOWLEDGMENT}

The authors thank A. Fauquet and A. Moreno for the polishing and cutting of the samples. 


\section{REFERENCES}

[1] R. P. H. Kooyman and L. M. Lechuga, "Immunosensors based on total internal reflectance," in Handbook of Biosensors and Electronic Noses, E. Kress-Rogers, Ed. Boca Raton, FL: CRC, 1997], pp. 169-196.

[2] D. Clerc and W. Lukosz, "Direct immunosensign with an integratedoptical grating coupler," Sens. Actuators B, Chem., vol. 40, pp. 53-58, 1997.

[3] C. Fattinger, H. Koller, D. Schlatter, and P. Wehrli, "The difference interferometer: A highly sensitive optical probe for quantification of molecular surface concentration," Biosen. Biolectron., vol. 8, pp. 99-107, 1993.

[4] R. Cush, J. M. Cronin, J. W. Stewart, C. H. Maule, J. Molloy, and N. J. Goddard, "The resonant mirror: A novel optical biosensor for direct sensing of biomolecular interactions-Part I: Principle of operation and associated instrumentation," Biosen. Biolectron., vol. 8, pp. 347-353, 1993.

[5] R. C. Alfernes, Guided Wave Optoelectronics, T. Tamir, Ed. Berlin, Germany: Springer-Verlag, 1988, ch. Chapter 4.

[6] W. Lukosz, "Principles and sensitivities of integrated optical and surface plasmon sensors for direct affinity sensing and immunosensing," Biosen. Biolectron., vol. 6, pp. 215-225, 1991.

[7] M. Weisser, G. Tovar, S. Mittler-Neher, W. Knoll, F. Brosinger, H Freimuth, M. Lacher, and W. Ehrfeld, "Specific bio-recognition reactions observed with an integrated Mach-Zehnder Interferometer," Biosen. Biolectron., vol. 14, pp. 405-411, 1999.

[8] K. Tiefenthaler and W. Lukosz, "Sensitivity of grating couplers as integrated-optical chemical sensors," J. Opt. Soc. Amer. B, Opt. Phys., vol. 6, pp. 209-220, 1989.

[9] O. Parriaux and G. J. Veldhuis, "Normalized analysis for the sensitivity optimization of integrated optical evanescent-wave sensors," J. Lightwave Technol., vol. 16, pp. 573-582, 1998.

[10] L. M. Lechuga, A. T. M. Lenferink, R. P. H. Kooyman, and J. Greve, "Feasibility of evanescent wave interferometer immunosensors for direct detection of pesticides: Chemical aspects," Sens. Actuators B, Chem., vol. 24-25, p. 762, 1995.

[11] E. F. Shipper, A. M. Brugman, C. Domínguez, L. M. Lechuga, R. P H. Kooyman, and J. Greve, "The realization of an integrated MachZehnder waveguide immunosensor in silicon technology," Sens. Actuators B, Chem., vol. 40, pp. 147-153, 1997.

[12] F. Prieto, A. Lobera, D. Jiménez, C. Domínguez, A. Calle, and L. M Lechuga, "Design and analysis of silicon antiresonant reflecting optical waveguides for evanescent field sensors," J. Lightwave Technol., vol. 18, pp. 966-972, 2000

[13] M. A. Duguay, Y. Kokubun, and T. L. Koch, "Antiresonant reflecting optical waveguides in SiO2-Si multilayer structures," Appl. Phys. Lett., vol. 49, pp. 13-15, July 1986.

[14] T. Baba and Y. Kokubun, "Dispersion and radiation loss characteristics of antiresonant reflecting optical waveguides: numerical results and analytical espressions," IEEE J. Quantum Electron., vol. 28, pp. 1689-1700, 1992.
[15] E. Bartolomé, M. Moreno, J. Muñoz, and C. Domínguez, "Multilayer analysis of ARROW structures," Microw. Opt. Technol. Lett., vol. 10, 1995.

[16] T. L. Koch, E. G. Burkhardt, F. G. Storz, T. J. Bridges, and T. Siger, "Vertically grating-coupled ARROW structures for III-V integrated optics," IEEE J. Quantum Electron., vol. QE-23, pp. 889-897, 1987.

[17] T. Baba and Y. Kokubun, "New polarization insensitive antiresonant reflecting optical waveguide ARROW-B," IEEE Photon. Technol. Lett. vol. 1, pp. 232-234, 1989.

[18] C. M. Kim and R. V. Ramaswamy, "Modeling of graded-index channel waveguides using nonuniform finite difference method," J. Lightwave Technol., vol. 7, pp. 1581-1589, 1989.

[19] I. Garcés, F. Villuendas, J. A. Vallés, C. Domínguez, and M. Moreno, "Analysis of leakage properties and guiding conditions of rib antiresonant reflecting optical waveguides," J. Lightwave Technol., vol. 14, pp. 798-805, 1996.

[20] M. Moreno, I. Garcés, J. Muñoz, C. Domínguez, J. Calderer, F. Villuendas, and J. Pelayo, "CMOS compatible ARROW guides," in Proc. 8th CIMTEC, Florence, Italy, 1994, S-VII.2.

[21] C. Domínguez, J. A. Rodríguez, F. J. Muñoz, and N. Zine, "Plasma enhanced CVD silicon oxide films for integrated optic applications," Vacuum, vol. 52, pp. 395-400, 1999

[22] G. R. Quigley, R. D. Harris, and J. S. Wilkinson, "Sensitivity enhancement of integrated optical sensors by use of thin high-index films," Appl. Opt., vol. 38, no. 28, pp. 6036-6039, Oct. 1999.

F. Prieto, photograph and biography not available at the time of publication.

L. M. Lechuga, photograph and biography not available at the time of publication.

A. Calle, photograph and biography not available at the time of publication.

A. Llobera, photograph and biography not available at the time of publication

C. Domínguez, photograph and biography not available at the time of publication. 